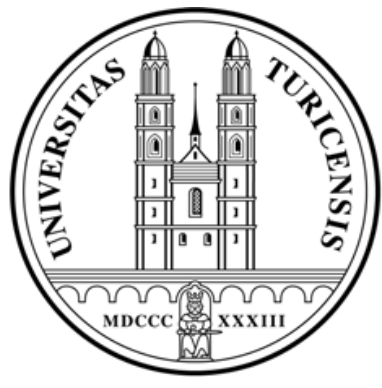

Institute for Empirical Research in Economics

University of Zurich

Working Paper Series

ISSN 1424-0459

Working Paper No. 208

Contest success function with the possibility of a draw:

axiomatization

Pavlo Blavatskyy

October 2004 


\title{
Contest success function with the possibility of a draw: axiomatization
}

\author{
Pavlo Blavatskyy* \\ Institute for Empirical Research in Economics \\ University of Zurich \\ Winterthurerstrasse 30 \\ CH-8006 Zurich \\ Switzerland \\ Phone: $+41(1) 6343586$ \\ Fax: $+41(1) 6344978$ \\ e-mail: pavlo.blavatskyy@,iew.unizh.ch
}

October 2004

\begin{abstract}
:
In imperfectly discriminating contests the contestants contribute effort to win a prize but the highest contributed effort does not necessarily secure a win. The contest success function (CSF) is the technology that translates an individual's effort into his or her probability of winning. This paper provides an axiomatization of CSF when there is the possibility of a draw (the sum of winning probabilities across all contestants is non-additive).
\end{abstract}

Keywords: contest success function, draw, logit, irrelevant alternatives, imperfectly

discriminating

JEL Classification codes: C72, D72, L13

* I am grateful to Patrick Leoni, and Stergios Skaperdas for helpful comments. Ganna Pogrebna helped a lot with English copy editing. 


\section{Contest success function with the possibility of a draw: axiomatization}

\section{Introduction}

A contest is a competition where the contestants simultaneously contribute effort to win a prize. All contests can be classified as either perfectly or imperfectly discriminating (e.g. Hillman and Riley 1989). In perfectly discriminating contests (e.g. Moldovanu and Sela 2001) the highest contributed effort secures a win (like in an all-pay auction). In imperfectly discriminating contests (e.g. Dixit 1987) the highest contributed effort has the highest probability of a win, but it does not necessarily secure a win. Imperfectly discriminating contests are extensively used to study sport competitions (e.g. Szymanski 2003), political rent-seeking (e.g. Nitzan 1991, 1994), research and development and patent races (e.g. Nti 1997), labor incentives (e.g. Rosen 1986) to name a few.

In imperfectly discriminating contests the technology that translates an individual's effort into his or her probability of winning is called the contest success function (CSF). The majority of studies on imperfectly discriminating contests use the logit CSF introduced by Tullock (1980) and subsequently axiomatized by Skaperdas (1996) and Clark and Riis (1996, 1998). In some studies (e.g. Hirshleifer 1989, Baik 2004) the logit CSF is called the ratio-form CSF. Other forms of CSF include the probit CSF (e.g. Lazear and Rosen 1981, Dixit 1987) and the difference-form CSF (e.g. Hirshleifer 1989). All existing forms of CSF share one limitation. The sum of winning probabilities across all contestants is assumed to be additive to unity. Thus, only one result is admitted in the contest — one of the contestants wins the prize unilaterally.

The majority of real life contests admit the possibility of ending without either side winning, which will be further referred to as a draw. A draw is a situation when two or more contestants obtain equal rights for the prize and they share the price. The possibility of a draw is 
apparent in sport tournaments (e.g. chess, football), political rent-seeking (e.g. no party wins the majority and a winning coalition is formed), research and development and patent races (e.g. a simultaneous independent discovery), labor tournaments (e.g. two individuals are promoted to share a senior position), military conflicts (e.g. no party can force a win and a cease-fire or peace agreement is reached). To analyze such imperfectly discriminating contests the most appropriate tool is a CSF that admits the possibility of a draw. This paper presents the axiomatic characterization of such CSF. This axiomatization is a generalization of Skaperdas (1996) and Clark and Riis (1998) when the sum of winning probabilities across all contestants is nonadditive.

The remainder of this paper is organized as follows. The logical exposition and the intuition behind the proposed system of axioms is presented in section two. This section describes the simplest case when there are only two contestants. In section three, which is more technical, a general result for any number of contestants is presented. Section four concludes.

\section{Contest with two contestants}

Consider first the simplest contest with only two contestants. This simple contest is of a special interest because:

- a draw is most probable to occur in pairwise contests,

- the model of a pairwise contest is extensively used in the literature (e.g. Rosen 1986, Baye and Shin 1999, Gradstein and Konrad 1999, Morgan 2003, Baik 2004), and

- in a special case when there are only two contestants it is possible to generalize the existing axiomatization by Skaperdas (1996) and Clark and Riis (1998) by relaxing their axioms of additivity and homogeneity. 
In what follows, the notation as in Skaperdas (1996) and Clark and Riis (1998) will be used wherever possible. Let $p^{i}\left(y_{1}, y_{2}\right) \in[0,1]$ be the probability that the $i$-th player wins the contest, $i \in[1,2]$, when the first (second) contestant contributes effort $y_{1} \geq 0 \quad\left(y_{2} \geq 0\right) .{ }^{1}$ Let $p^{12}\left(y_{1}, y_{2}\right) \in[0,1]$ be the probability that the first and the second player end up in a draw.

Axiom 1 (Additivity) $p^{1}\left(y_{1}, y_{2}\right)+p^{2}\left(y_{1}, y_{2}\right)+p^{12}\left(y_{1}, y_{2}\right)=1, \forall y_{1}, y_{2} \geq 0$

Axiom 2 (Nontriviality) $p^{1}(0,)=0,. p^{2}(., 0)=0, p^{12}(0,0)=1$ and $d p^{1}\left(y_{1}, 0\right) / d y_{1}>0$, $d p^{2}\left(0, y_{2}\right) / d y_{2}>0$

Axioms 1 and 2 guarantee that the winning probabilities of two contestants together with their probability of a draw indeed constitute a non-trivial probability distribution. When one of the contestants contributes zero effort he or she cannot win the contest: but, he or she can end up in a draw. When two contestants contribute zero effort they end up in a draw with certainty. For every contestant the probability of winning is increasing in his or her own effort when the opponent contributes zero effort. $^{2}$

Axiom 3 (Independence of irrelevant alternatives) The propability that the $i$-th contestant wins given that the $j$-th contestant does not win depends only on the effort level of the $i$-th contestant (and it does not depend on the effort level of the $j$-th contestant), $i, j \in[1,2], i \neq j$.

Theorem 1 Axioms 1-3 hold if and only if $p^{1}\left(y_{1}, y_{2}\right)=\frac{H^{1}\left(y_{1}\right)}{1+H^{1}\left(y_{1}\right)+H^{2}\left(y_{2}\right)}$, $p^{2}\left(y_{1}, y_{2}\right)=\frac{H^{2}\left(y_{2}\right)}{1+H^{1}\left(y_{1}\right)+H^{2}\left(y_{2}\right)}$ and $p^{12}\left(y_{1}, y_{2}\right)=\frac{1}{1+H^{1}\left(y_{1}\right)+H^{2}\left(y_{2}\right)}$

\footnotetext{
${ }^{1}$ Since the event of a draw is explicitly allowed, there is no problem in considering a zero effort of the contestnats. Skaperdas (1996) and Clark and Riis (1998) considered only strictly positive effort levels because without the possibility of a draw, the CSF exhibits a discontinuous jump when all contestants contribute zero effort.

${ }^{2}$ Clark and Riis (1998) assumed in addition that for every contestant the probability of winning is nonincreasing in his or her opponent's effort, $\partial p^{i}\left(y_{1}, y_{2}\right) / \partial y_{j} \leq 0, i, j \in[1,2], i \neq j$. This property follows from the conjunction of axioms 1-3 and there is no need to assume it explicitly.
} 
where function $H^{i}\left(y_{i}\right)$ is strictly increasing i.e. $d H^{i}\left(y_{i}\right) / d y_{i}>0$ and $H^{i}(0)=0, i \in[1,2]$.

Proof of this and all subsequent theorems is presented in Appendix.

Given axioms 1-3, theorem 1 guarantees that for every contestant the probability of winning is increasing in his or her effort and decreasing in the opponent's effort. For every contestant the probability of a draw is decreasing both in his or her effort and in the opponent's effort. Thus, when both contestants contribute zero effort they end up in a draw with certainty but the higher the effort levels that they contribute, the less probable a draw is to occur. This property reflects an intuitive observation that in real-life contests a draw is more (less) probable when the contestants contribute low (high) effort on average.

For example, in football it is more common to observe draws with low scores (e.g. " $0: 0$ ", "1:1") than those with high scores (e.g. "2:2", "3:3"). This can be interpreted that when both teams put a lot of effort into the game they are less likely to end up in a draw. During an elimination tournament the ending of a football match is restricted and a draw is not allowed. If neither team can reach a victory within a normal time limit both teams are required to play an extra added time until one side wins. It can also be interpreted that such requirement results in the contribution of higher effort by both teams making a draw less likely to occur during the additional time. According to the CSF described in theorem 1 the probability of a draw is converging to zero when both the first and the second contestant contribute high effort. Thus, for high effort levels the CSF described in theorem 1 converges to the logit CSF without the possibility of a draw (e.g. Skaperdas 1996).

Theorem 1 also implies that, for example, when the first player contributes zero effort, the second player does not automatically win the contest. In such a case the most probable outcome of a contest is either a draw (when the second contestant contributes low effort) or a win for the 
second player (if he or she contributes high effort). More generally, the CSF implied by theorem 1 does not allow any contestant to secure a win with certainty. The logit CSF without the possibility of a draw (e.g. Tullock 1980) allows a contestant to secure a win with certainty in a special case when all his or her oponents contribute zero effort.

Axiom 4 (homogeneity) $\frac{P^{1}\left(y_{1}, y_{2}\right)}{P^{2}\left(y_{1}, y_{2}\right)}=\frac{P^{1}\left(\lambda \cdot y_{1}, \lambda \cdot y_{2}\right)}{P^{2}\left(\lambda \cdot y_{1}, \lambda \cdot y_{2}\right)}, \forall \lambda>0, \forall y_{1}, y_{2}>0$

According to axiom 4 the ratio of winning probabilities remains unchanged if both contestants augment their effort by the same amount. Skaperdas (1996) and Clark and Riis (1998) formulated the homogeneity axiom in absolute terms i.e. $P^{i}\left(y_{1}, y_{2}\right)=P^{i}\left(\lambda \cdot y_{1}, \lambda \cdot y_{2}\right), \forall \lambda>0$, $i \in[1,2]$. However, when a draw is allowed, as shown above, the probability of a draw decreases when both contestants augment their effort. In absolute terms the sum of winning probabilities increases when both contestants augment their effort. Therefore, the homogeneity axiom does not hold in absolute terms but it can be assumed that it holds in relative terms.

Theorem 2 Axioms 1-4 hold if and only if $p^{i}\left(y_{1}, y_{2}\right)=\frac{\alpha_{i} y_{i}^{r}}{1+\alpha_{1} y_{1}^{r}+\alpha_{2} y_{2}^{r}}$ and $p^{12}\left(y_{1}, y_{2}\right)=\frac{1}{1+\alpha_{1} y_{1}^{r}+\alpha_{2} y_{2}^{r}}$, where $r, \alpha_{i}>0$ are constant, $i \in[1,2]$

Axiom 4 assumes that the relative winning probabilities are homogeneous of degree zero. The consequence of this assumption is that the strictly increasing function $H^{i}\left(y_{i}\right)$ introduced in theorem 1 is uniquely specified. In particular, function $H^{i}\left(y_{i}\right)$ is a power function. Common to all contestants, constant $r$ captures the marginal increase in a winning probability caused by a higher effort. Contests with low $r$ can be regarded as poorly discriminating or "noisy" contests (e.g. Blavatskyy 2004). When $r$ converges to zero the contest outcome converges to a random lottery with no dependence on the contributed efforts of the contestants. Contests with high $r$ can 
be regarded as highly discriminating and when $r$ converges to infinity, the contest outcome is determined by an all-pay auction (e.g. Moldovanu and Sela 2001). Clark and Riis (1998) provided an intuitive explanation of an individual specific constant $\alpha_{i}$ as a degree of unfairness inside the contest. The higher the constant $\alpha_{i}$ the more probable is the $i$-th contestant to win given that he or she submits the same effort as the opponents.

Skaperdas (1996) proposed as an anonymity axiom that individual characteristics are irrelevant for the CSF (only individual efforts matter), which could be formally expressed as $p^{1}\left(y_{1}, y_{2}\right)=p^{2}\left(y_{2}, y_{1}\right)$. The anonymity axiom in conjunction with axiom 1 immediately implies that the probability of a draw is symmetric i.e. $p^{12}\left(y_{1}, y_{2}\right)=p^{12}\left(y_{2}, y_{1}\right)$. When the anonymity axiom is added to axioms 1-3 (axioms 1-4) we obtain theorem 1 (theorem 2) with $H^{1}()=.H^{2}($. ( $\alpha_{1}=\alpha_{2}=\alpha$ ). Notice that when $\alpha$ converges to infinity the probability of a draw converges to zero and we obtain the logit CSF without the possibility of a draw $p^{i}\left(y_{1}, y_{2}\right)=y_{i}^{r} /\left(y_{1}^{r}+y_{2}^{r}\right)$, $i \in[1,2]$, axiomatized by Skaperdas (1996). When $\alpha$ converges to zero the contest ends up in a draw with certainty. Thus, we can interpret parameter $\alpha$ as a relative likelihood of a draw in the contest.

\section{Contest with $n>2$ contestants}

Let $N=\{1, \ldots, n\}$ denote the set of all contestants and $\Omega$ be the set of all subsets in $N$. For any group of contestants $S \subseteq \Omega$ let $|S|$ denote the number of contestants in this group. The $i$-th contestant contributes effort $y_{i} \geq 0, i \in N$. For any group of contestants $S=\left\{i_{1}, \ldots, i_{|S|}\right\} \subseteq \Omega$ let us denote by $y_{S}=\left(y_{i_{1}}, \ldots, y_{i_{|S|}}\right)$ the vector of efforts contributed by the contestants grouped in $S$. Note that $y_{\{i\}}=y_{i}, \forall i \in N$ and $y_{N}=\left(y_{1}, \ldots, y_{n}\right)$. Finally, denote by $p^{S}\left(y_{N}\right) \in[0,1]$ the probability that 
a group $S \subseteq \Omega$ of contestants ends up in a draw and all remaining contestants i.e. $N \backslash \mathrm{S}$ loose the contest. The probability that the $i$-th contestant wins the contest unilaterally is defined as $p^{\{i\}}\left(y_{N}\right), i \in N$. Axioms 1a-3a are the generalization of axioms 1-3 for the contest with $n>2$ contestants.

$$
\text { Axiom 1a } \sum_{S \subseteq \Omega} p^{S}\left(y_{N}\right)=1, \forall y_{N} \in \mathrm{R}_{+}^{n}
$$

When more than two contestants participate in the contest, first of all, all $n$ contestants can end up in a draw together. Additionally any group of $n-1, n-2$ etc contestants can end up in a draw. Finally, any one of $n$ contestants can win the contest unilaterally. These events are mutually exclusive: only one of them is true ex post. Therefore, axiom 1a requires the probability distribution over all possible groups of contestants to be additive to unity.

$$
\begin{aligned}
& \text { Axiom 2a } \forall S \subseteq \Omega, S \neq N: p^{S}\left(y_{N}\right)=0 \text { if } y_{i}=0, \forall i \in S ; p^{N}(0)=1 \text { and } \\
& d P^{\{i\}}\left(0, \ldots, y_{i}, \ldots, 0\right) / d y_{i}>0, \forall i \in N
\end{aligned}
$$

According to axiom 2a if there is any group of, but not all, contestants such that all its members contribute zero effort, then the members of this group cannot end up in a draw only with each other. Axiom 2a implies inter alia that a contestant who contributes zero effort cannot win the contest. If all contestants contribute zero effort all of them end up in a draw with certainty.

$$
\text { Axiom 3a } \forall S \subseteq \Omega: \frac{p^{S}\left(y_{N}\right)}{1-\sum_{\substack{T \subseteq \Omega \\ S \nsubseteq T}} p^{T}\left(y_{N}\right)}=f^{S}\left(y_{S}\right) \text { where } f^{S}: \mathrm{R}_{+}^{|S|} \rightarrow[0,1] \text { is an arbitrary }
$$

function

The members of a group $S$ are successful if all of them end up in a draw either with each other or together with the contestants that are not members of group $S$. According to axiom 3 a the 
probability that a group $S$ ends up in a draw, conditional on the success of all its members, depends only on the efforts of its members and it does not depend on the efforts of other contestants that are not members of the group $S$.

Theorem 3 Axioms 1a-3a hold if and only if $p^{S}\left(y_{N}\right)=\frac{H^{S}\left(y_{S}\right) \sum_{\substack{A \subseteq \Omega \\ S \subseteq A}} H^{A}\left(y_{A}\right) \sum_{\substack{B \subseteq \Omega \\ A \subset B}} H^{B}\left(y_{B}\right) \ldots H^{N}\left(y_{N}\right)}{\sum_{A \subseteq \Omega} H^{A}\left(y_{A}\right) \sum_{\substack{B \subseteq \Omega \\ A \subset B}} H^{B}\left(y_{B}\right) \ldots H^{N}\left(y_{N}\right)}$ where $H^{N}\left(y_{N}\right)=1$, and for any group $S \subset \Omega$ function $H^{S}\left(y_{S}\right)$ is strictly increasing i.e. $\partial H^{S}\left(y_{S}\right) / \partial y_{i}>0, \forall i \in S$, and $H^{S}(0)=0$

According to theorem 3 axioms 1a-3a hold if and only if the CSF has the following structure. When all $n$ contestants contribute zero effort, all of them end up in a draw with certainty. However, the probability of such ending $p^{N}\left(y_{N}\right)=\frac{1}{\left.\sum_{\substack{A \subseteq \Omega \\ H^{A}}} H_{A}\right) \sum_{\substack{B \subseteq \Omega \\ A \subset B}} H^{B}\left(y_{B}\right) \ldots H^{N}\left(y_{N}\right)}$ strictly decreases in every contestant's effort i.e. $\partial p^{N}\left(y_{N}\right) / \partial y_{i}<0, \forall i \in N$. Now consider a group $N \backslash\{i\}$ of all contestants except for the $i$-th contestant, $i \in N$. When all members of the group $N \backslash\{i\}$ contribute zero effort, they cannot end up in a draw only with each other. In general the probability of such ending $p^{N\{i\}}\left(y_{N}\right)$, when compared with the probability $p^{N}\left(y_{N}\right)$, is a strictly increasing function of the efforts of every member of the group $N \backslash\{i\}$ i.e. $p^{N \backslash\{i\}}\left(y_{N}\right)=H^{N \backslash\{i\}}\left(y_{N \backslash\{i\}}\right) \cdot p^{N}\left(y_{N}\right)$. Thus, when the members of group $N \backslash\{i\}$ contribute higher effort the ending when the group $N \backslash\{i\}$ shares the prize becomes relatively more probable than the ending when all contestants share the prize.

Now consider a group $N \backslash\{i, j\}$ of all contestants except for the $i$-th and the $j$-th contestant, $i, j \in N, i \neq j$. When compared with the probability $p^{N}\left(y_{N}\right)+p^{N \backslash\{i\}}\left(y_{N}\right)+p^{N \backslash\{j\}}\left(y_{N}\right)$ the 
probability that the members of the group $N \backslash\{i, j\}$ end up in a draw only with each other is a strictly increasing function of the efforts of every member of this group. Intuitively, every member of the group $N \backslash\{i, j\}$ is also a member of the larger groups $N \backslash\{i\}$ and $N \backslash\{j\}$. Thus, higher efforts of the members of the group $N \backslash\{i, j\}$ automatically increase the relative likelihood that the groups $N \backslash\{i\}$ and $N \backslash\{j\}$ share the prize. According to theorem 3 when the members of the group $N \backslash\{i, j\}$ contribute higher effort the ending when the group $N \backslash\{i, j\}$ shares the prize becomes relatively more probable than the (also more probable) ending when the groups $N \backslash\{i\}$, $N \backslash\{j\}$ and $N$ share the prize. Formally, this corresponds to $p^{N \backslash\{i, j\}}\left(y_{N}\right)=H^{N \backslash\{i, j\}}\left(y_{N \backslash\{i, j\}}\right) \cdot\left(1+H^{N \backslash\{i\}}\left(y_{N \backslash\{i\}}\right)+H^{N \backslash\{j\}}\left(y_{N \backslash\{j\}}\right)\right) \cdot p^{N}\left(y_{N}\right)$. The same logical structure of the CSF is preserved for all other groups of contestants.

It turns out that when more than two contestants participate in the contest the axiom of homogeneity as in Skaperdas (1996) and Clark and Riis (1998) cannot be generalized without imposing a restriction of the structure of contest outcome (ending). Specifically the homogeneity of relative winning probabilities (axiom 4a below) is compatible with theorem 3 only when the contest with more than two players can end up either with one player winning unilaterally or a group of players of a fixed size sharing the prize. An example of such restriction is when either only two, or only three or only all contestants together can end up in a draw ${ }^{3}$. Thus, $n=2$ is the only special case when the homogeneity axiom does not restrict the structure of the contest outcome (ending).

Intuitively, an individual's low effort yields a relatively high economic benefit when the overall aggregate effort is low. In such case, besides a unilateral victory, there is also a high probability of a draw with the opponents who contributed high effort. However, when every

\footnotetext{
${ }^{3}$ An example when only all contestants together can end up in a draw is analyzed in section 3.2 below.
} 
contestant augments his or her effort by the same amount the overall aggregate effort contributed in the contest increases. In this case, the relative economic benefit of an individual's low effort decreases. A low effort yields only a chance of unilateral victory. The probability of a draw with the opponents who contributed high effort converges to zero. Thus, the homogeneity of winning probabilities does not hold because at low aggregate effort the possibility of a draw dilutes the economic benefit of effort differentials. One possibility to overcome this problem is to assume that the homogeneity of winning probabilities is preserved in the limit when all contestants contribute infinitely high effort. However, this assumption does not allow to specify the monotone functions that define the probabilities of a draw.

\subsection{Example of the CSF when a contest outcome is unrestricted}

When we keep the assumption that any group $S \subseteq \Omega$ of contestants can share the prize,

the CSF can be formulated solely in general monotone functions $H^{S}\left(y_{S}\right)$ as in theorem 3. Figures 1 and 2 demonstrate the CSF from theorem 3 when there are three contestants and $H^{S}\left(y_{S}\right)=\left(\sum_{i \in S} y_{i}\right)^{2}$. Figure $1(2)$ demonstrates the probability of a win and a draw for the first contestant when his or her opponents contribute effort $y_{2}=y_{3}=0\left(y_{2}=0.5, y_{3}=0.4\right)$. When the first contestant contributes low effort $\left(y_{1} \rightarrow 0\right)$ the most probable successful ending of the contest for the first contestant is a draw together with two opponents. If the first contestant does not end up in a draw with two opponents while contributing low effort he or she is more probable to end up in a draw with one opponent who submitted positive effort rather than to win the contest unilaterally (e.g. figure 2).

When the first contestant contributes high effort $\left(y_{1} \rightarrow+\infty\right)$ the most probable ending of the contest is that the first contestant wins the prize unilaterally. If the first contestant does not 
win while contributing high effort he or she is more probable to end up in a draw with one opponent rather than in a draw with both opponents. A draw with an opponent who contributed higher effort is more probable that a draw with an opponent who contributed lower effort. When one of the opponents contributes high effort a draw with such an opponent can become the most probable successful ending for the contestant who contributes low or moderate effort (e.g. figure 2 when $\left.y_{1}=0.6\right)$. Figures 1 and 2 also demonstrate that:

- the probability that the first player wins the contest is strictly increasing in his or her effort,

- the probability that the first contestant ends up in a draw with one of the opponents is first increasing and then decreasing in his or her effort, and

- the probability that the first contestant ends up in a draw with all opponents is strictly decreasing in his or her effort. 


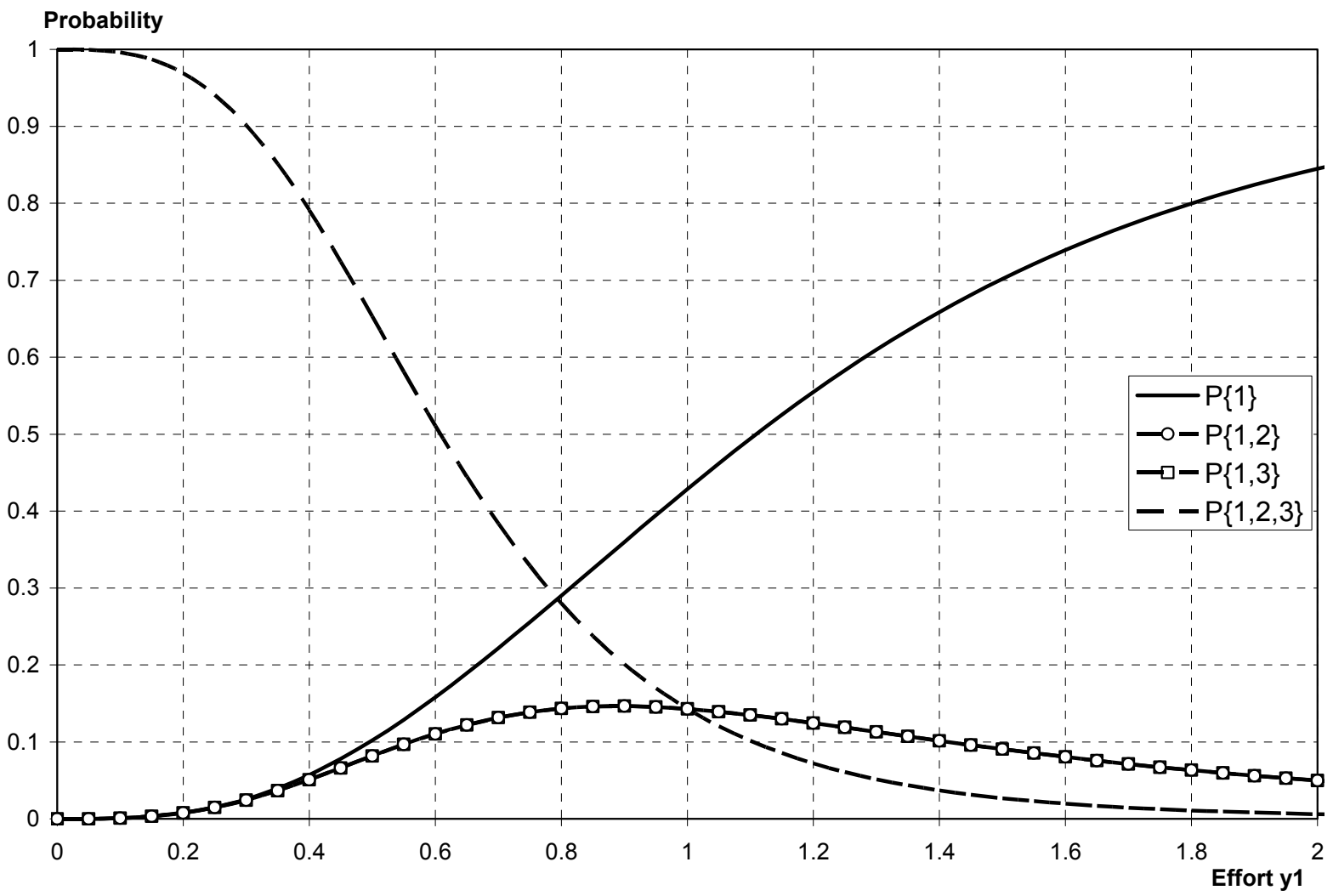

Figure 1 Probability $p^{\{1\}}\left(p^{\{1,2\}}, p^{\{1,3\}}, p^{\{1,2,3\}}\right)$ that the first contestant wins in the contest with three contestants (ends up in a draw with the second, the third, the second and the third contestant) while contributing effort $y_{1}\left(y_{2}=y_{3}=0\right)$ 


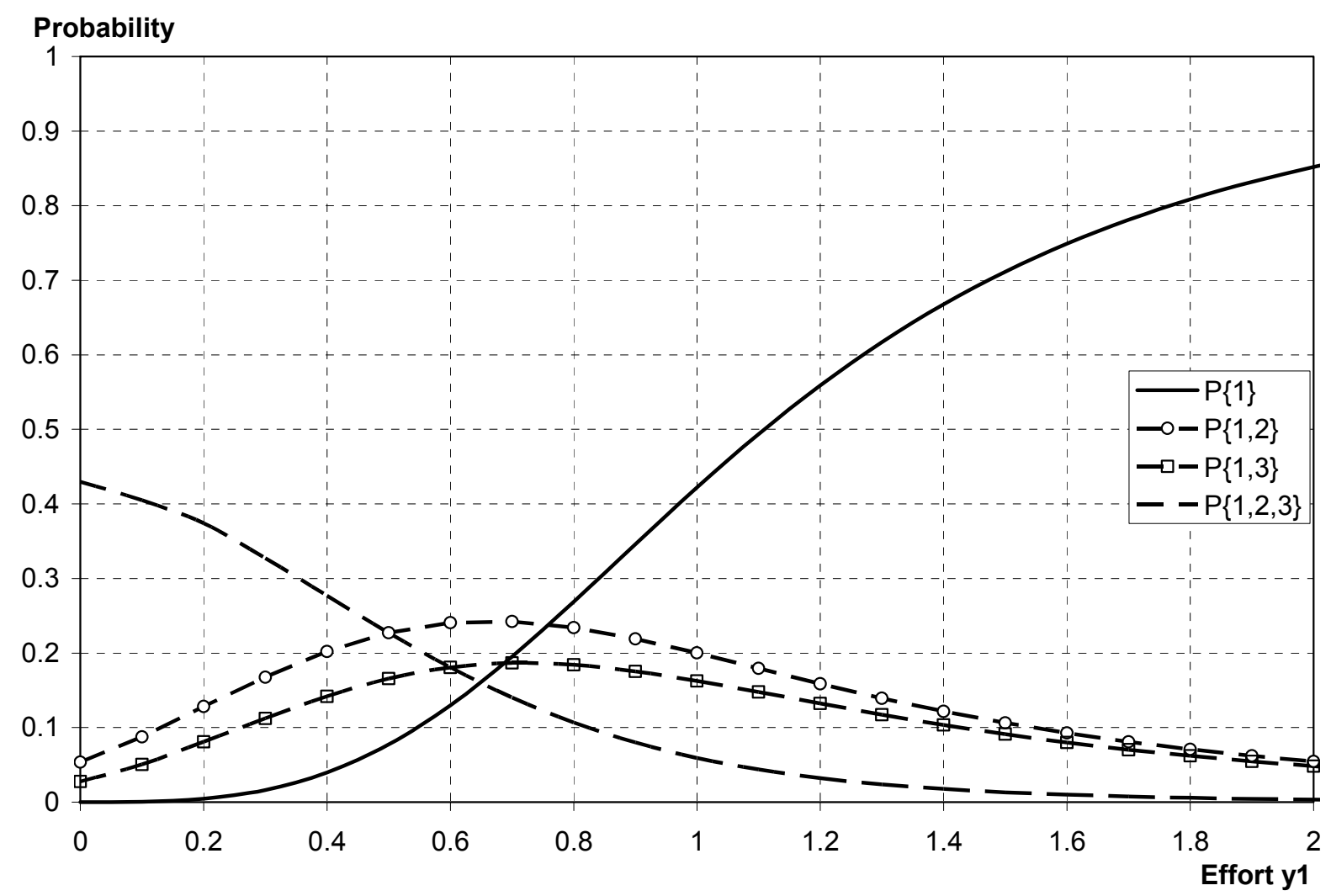

Figure 2 Probability $p^{\{1\}}\left(p^{\{1,2\}}, p^{\{1,3\}}, p^{\{1,2,3\}}\right)$ that the first contestant wins in the contest with three contestants (ends up in a draw with the second, the third, the second and the third contestant) while contributing effort $y_{1}\left(y_{2}=0.5, y_{3}=0.4\right)$ 


\subsection{Example of the CSF when a contest outcome is restricted}

Consider a special case when a contest outcome is restricted so that either one of $n$ contestants wins the contest unilaterally or all contestants end up in a draw together. This kind of a draw can be interpreted as a stalemate when competition ends up in a deadlock with none of the contestants being able to force a unilateral win. Formally, a restriction $p^{S}\left(y_{N}\right)=0$ is imposed for every group $S \subset \Omega, S \neq\{i\}, i \in N$. This restriction allows to simplify theorem 3 into corollary 1 below and to impose homogeneity on the relative winning probabilities as done in axiom 4a below.

Corollary $1 \forall S \subset \Omega, S \neq\{i\}: p^{S}\left(y_{N}\right)=0 \Rightarrow$ axioms 1a-3a hold if and only if $p^{\{i\}}\left(y_{N}\right)=\frac{H^{i}\left(y_{i}\right)}{1+\sum_{j=1}^{n} H^{j}\left(y_{j}\right)}$ and $p^{N}\left(y_{N}\right)=\frac{1}{1+\sum_{j=1}^{n} H^{j}\left(y_{j}\right)}$ where function $H^{i}\left(y_{i}\right)$ is strictly increasing i.e. $\partial H^{i}\left(y_{i}\right) / \partial y_{i}>0$ and $H^{i}(0)=0, \forall i \in N$

Proof of corollary 1 follows immediately from theorem 3

$$
\text { Axiom } 4 \mathbf{a} \frac{P^{i}\left(y_{N}\right)}{P^{j}\left(y_{N}\right)}=\frac{P^{i}\left(\lambda \cdot y_{N}\right)}{P^{j}\left(\lambda \cdot y_{N}\right)}, \forall \lambda>0, \forall i, j \in N, y_{i}, y_{j} \neq 0
$$

Axiom 4a is sufficiently restrictive to specify uniquely the monotone functions $H^{i}\left(y_{i}\right)$ from corollary 1. Specifically, axiom 4 a holds if and only if functions $H^{i}\left(y_{i}\right)$ are power functions (corollary 2)

Corollary $2 \forall S \subset \Omega, S \neq\{i\}: p^{S}\left(y_{N}\right)=0 \Rightarrow$ axioms 1a-4a hold if and only if $p^{\{i\}}\left(y_{N}\right)=\frac{\alpha_{i} y_{i}^{r}}{1+\sum_{j=1}^{n} \alpha_{j} y_{j}^{r}}$ and $p^{N}\left(y_{N}\right)=\frac{1}{1+\sum_{j=1}^{n} \alpha_{j} y_{j}^{r}}$ where $r, \alpha_{i}>0$ are constant, $i \in N$ 
Proof of corollary 2 is analogous to the proof of theorem 2 with axioms 1a-4a and corollary 1 being used instead of axioms $1-4$ and theorem 1 correspondingly.

The CSF from corollary 2 generically applies to "unfair" contests (e.g. Clark and Riis, 1998). In "unfair" contests the contestants are heterogeneous: the same effort yields different probability of winning when it is contributed by different contestants. The anonymity axiom as in Skaperdas (1996) imposes a restriction that a contest is fair i.e. two different contestants have the same probability of winning when they contribute the same effort. Formally, this corresponds to $p^{\{i\}}\left(y_{N}\right)=p^{\{j\}}\left(y_{N}\right)$ when $y_{i}=y_{j}, \quad \forall i, j \in N$. The anonymity axiom implies that the coefficients $\alpha_{i}>0$ from corollary 2 are identical for every contestant i.e. $\alpha_{i}=\alpha, \forall i \in N$

\section{Conclusion}

This paper presents an axiomatic characterization of the CSF with a rich structure on the outcome of the contest. Specifically, the possibility of a draw is allowed so that any group of the contestants can end up in a "shared victory" of the contest. In a special case of a simple pairwise contest it is possible to generalize the logit CSF axiomatized by Skaperdas (1996) and Clark and Riis (1998) to allow for the possibility of a draw. The obtained logit CSF with the possibility of a draw is mainly characterized by the properties of the independence of irrelevant alternatives and the relative homogeneity of winning probabilities. However, the property of homogeneity (either in relative or absolute winning probabilities) cannot be sustained in a general case when there are more than two contestants unless a restriction on the structure of contest outcome is imposed. An example of such restriction is when either only two or only three or only all contestants together can end up in a draw.

The logit CSF with the possibility of a draw is shown to possess the following properties. When all contestants contribute high effort the logit CSF with the possibility of a draw converges 
to the logit CSF without the possibility of a draw. The probability that all contestants end up in a draw is the highest and equal to unity when all contestants contribute zero effort and it strictly decreases in the effort of every contestant. The probability that any group of two or more, but not all, contestants shares the prize increases in low effort of its members and decreases in high effort of its members. The probability that any contestant wins the contest unilaterally strictly increases in his or her effort.

The CSF with the possibility of a draw is a useful model in many economic situations where the ending of competition without either side winning is a plausible outcome. The CSF axiomatized in this paper can be employed instead the conventional logit CSF to analyze the paradoxical results in the literature on the imperfectly discriminating contests such as Tullock (1980) "overdissipation of rents" or the non-existence of pure strategy Nash equilibrium in highly discriminating contests (e.g. Blavatskyy 2004). The CSF with the possibility of a draw is continuous at zero and therefore it is more convenient as an analytical tool than the conventional logit CSF, which is discontinuous at zero. However, the CSF with a possibility of a draw is derived only in general monotone functions when more than two contestants participate in the contest and the structure of contest outcome is unrestricted. A useful extension of this work is to find an intuitively appealing property that allows to specify these monotone functions while preserving the rich structure of the contest outcome. 


\section{References}

Baik, K. (2004) "Two-player asymmetric contests with ratio-form contest success functions" Economic Inquiry, 42, 679-689

Baye, M. and Shin, O. (1999) "Strategic behaviour in contests: comment" American Economic Review, 89, 691-694

Blavatskyy, P. (2004) "Why the Olympics have three prizes and not just one" IEW Working Paper \#200 http://www.iew.unizh.ch/wp/iewwp200.pdf

Clark, D. and Riss, C. (1996) "On the win probability in rent-seeking games" Discussion Paper in Econoimcs E4/96, University of Tromso

Clark, D. and Riss, C. (1998) "Contest success functions: an extension" Economic Theory, 11, 201-204

Dixit, A. (1987) "Strategic behavior in contests" American Economic Review 77, 891-898

Gradstein, M. and Konrad, K. (1999) "Orchestrating rent seeking contests" The Economic Journal, 109, 536-545

Hillman, A. and Riley, J. (1989) "Politically contestable rents and transfers" Economics and Politics, 1, 17-39

Hirshleifer, J. (1989) "Conflict and rent-seeking success functions: ratio vs. difference models of relative success" Public Choice, 63, 101-112

Lazear, E. and Rosen, S. (1981) "Rank Order Tournaments as Optimal Labor Contracts" Journal of Political Economy, 89, 841-864

Moldovanu B. and Sela A. (2001) "The optimal Allocation of Prizes in Contests" American Economic Review, 91, 3, 542-558

Morgan, J. (2003) "Sequential contests” Public Choice, 116, 1-18

Nitzan, Sh. (1991) “Collective rent dissipation” The Economic Journal, 101, 1522-1534

Nitzan, Sh. (1994) "Modelling Rent-Seeking Contests," European Journal of Political Economy, $10,41-60$

Nti, K. (1997) "Comparative statics of contests and rent-seeking games" International Economic Review, 38, 43-59

Rosen, Sh. (1986) "Prizes and incentives in the elimination tournaments" American Economic Review, 76, 701-715

Skaperdas S. (1996) “Contest Success Functions”, Economic Theory, 7, 283-90

Szymanski S. (2003) "The Economic Design of Sporting Contests" Journal of Economic Literature, 41, 1137-1187 


\section{Appendix}

\section{Proof of theorem 1}

Let $H^{1}\left(y_{1}\right)=\frac{p^{1}\left(y_{1}, 0\right)}{1-p^{1}\left(y_{1}, 0\right)}$ and $H^{2}\left(y_{2}\right)=\frac{p^{2}\left(0, y_{2}\right)}{1-p^{2}\left(0, y_{2}\right)}$. Notice that this newly defined function $H^{i}\left(y_{i}\right)$ is strictly increasing i.e. $d H^{i}\left(y_{i}\right) / d y_{i}>0$ because according to axiom 2 $d p^{1}\left(y_{1}, 0\right) / d y_{1}>0$ and $d p^{2}\left(0, y_{2}\right) / d y_{2}>0$. Additionaly, $H^{i}(0)=0, i \in[1,2]$, due to axiom 2 as well. Axioms 2 and 3 together imply that $\frac{p^{1}\left(y_{1}, y_{2}\right)}{1-p^{2}\left(y_{1}, y_{2}\right)}=p^{1}\left(y_{1}, 0\right)$ and $\frac{p^{2}\left(y_{1}, y_{2}\right)}{1-p^{1}\left(y_{1}, y_{2}\right)}=p^{2}\left(0, y_{2}\right)$. Specifically, axiom 3 implies that the ratio $\frac{p^{1}\left(y_{1}, y_{2}\right)}{1-p^{2}\left(y_{1}, y_{2}\right)}$ does not depend on $y_{2}$ and therefore $\frac{p^{1}\left(y_{1}, y_{2}\right)}{1-p^{2}\left(y_{1}, y_{2}\right)}=\frac{p^{1}\left(y_{1}, 0\right)}{1-p^{2}\left(y_{1}, 0\right)}=p^{1}\left(y_{1}, 0\right)$. The last equality follows from axiom 2 which guarantees that $p^{2}\left(y_{1}, 0\right)=0$. Using the definition of function $H^{i}\left(y_{i}\right), i \in[1,2]$, we can write $p^{1}\left(y_{1}, 0\right)=H^{1}\left(y_{1}\right) /\left(1+H^{1}\left(y_{1}\right)\right)$ and $p^{2}\left(0, y_{2}\right)=H^{2}\left(y_{2}\right) /\left(1+H^{2}\left(y_{2}\right)\right)$. Axioms 2 and 3 then imply a system of equations (A1).

$$
\left\{\begin{array}{l}
\frac{p^{1}\left(y_{1}, y_{2}\right)}{1-p^{2}\left(y_{1}, y_{2}\right)}=\frac{H^{1}\left(y_{1}\right)}{1+H^{1}\left(y_{1}\right)} \\
\frac{p^{2}\left(y_{1}, y_{2}\right)}{1-p^{1}\left(y_{1}, y_{2}\right)}=\frac{H^{2}\left(y_{2}\right)}{1+H^{2}\left(y_{2}\right)}
\end{array}\right.
$$

The solution of system (A1) is $p^{1}\left(y_{1}, y_{2}\right)=\frac{H^{1}\left(y_{1}\right)}{1+H^{1}\left(y_{1}\right)+H^{2}\left(y_{2}\right)}, p^{2}\left(y_{1}, y_{2}\right)=\frac{H^{2}\left(y_{2}\right)}{1+H^{1}\left(y_{1}\right)+H^{2}\left(y_{2}\right)}$. From axiom 1 it follows that $p^{12}\left(y_{1}, y_{2}\right)=1-p^{1}\left(y_{1}, y_{2}\right)-p^{2}\left(y_{1}, y_{2}\right)=1 /\left(1+H^{1}\left(y_{1}\right)+H^{2}\left(y_{2}\right)\right)$. It is straightforward to verify that such functions $p^{1}\left(y_{1}, y_{2}\right), p^{2}\left(y_{1}, y_{2}\right)$ and $p^{12}\left(y_{1}, y_{2}\right)$ satisfy axioms 1-3. Thus, axioms 1-3 are both necessary and sufficient. Q.E.D. 


\section{Proof of theorem 2}

It follows from theorem 1 that $\frac{P^{1}\left(y_{1}, y_{2}\right)}{P^{2}\left(y_{1}, y_{2}\right)}=\frac{H^{1}\left(y_{1}\right)}{H^{2}\left(y_{2}\right)}$. Axiom 4 then is equivalent to the assumption that $\frac{H^{1}\left(y_{1}\right)}{H^{2}\left(y_{2}\right)}=\frac{H^{1}\left(\lambda \cdot y_{1}\right)}{H^{2}\left(\lambda \cdot y_{2}\right)}, \forall \lambda>0, \forall y_{1}, y_{2}>0$. Let $\lambda=1 / y_{2}$. We obtain then a functional equation

$$
H^{1}\left(y_{1} / y_{2}\right)=H^{2}(1) H^{1}\left(y_{1}\right) / H^{2}\left(y_{2}\right)
$$

Since equation (A2) holds for any positive $y_{2}$ by letting $y_{2}=y_{1}$ we establish that $H^{2}\left(y_{1}\right)=H^{2}(1) H^{1}\left(y_{1}\right) / H^{1}(1), \forall y_{1}>0$. Thus, we can rewrite (A2) as (A3):

$$
H^{1}\left(y_{1} / y_{2}\right)=H^{1}(1) H^{1}\left(y_{1}\right) / H^{1}\left(y_{2}\right)
$$

Let $y_{1}=e^{x+z}, y_{2}=e^{x}$ and $f(x)=\ln H^{1}\left(e^{x}\right)-\ln H^{1}(1)$. Notice that function $f($.$) is$ continuous because function $H^{1}()=.p^{1}(., 0) /\left(1-p^{1}(., 0)\right)$ is continuous. Equation (A3) then becomes Cauchy's functional equation $f(x+z)=f(x)+f(z)$, which has the unique solution $f(x)=r x$, where $r$ is constant. Using the definition of function $f($.$) we then obtain$ $H^{1}\left(y_{1}\right)=H^{1}(1) y_{1}^{r}$ and $H^{2}\left(y_{1}\right)=H^{2}(1) y_{1}^{r}, \forall y_{1}>0$. Since theorem 1 restricts $d H^{i}\left(y_{i}\right) / d y_{i}>0$ it must be the case that $r$ and $H^{i}(1)$ are non-zero and of the same sign, $i \in[1,2]$. Additionally, theorem 1 requires function $H^{i}($.$) to be strictly positive on \mathrm{R}_{++}$. Therefore, $r, H^{i}(1)>0$, $i \in[1,2]$. Finally, let $\alpha_{i}=H^{i}(1), i \in[1,2]$.

Substituting for the newly obtained function $H^{i}($.$) in theorem 1$ we obtain $p^{i}\left(y_{1}, y_{2}\right)=\frac{\alpha_{i} y_{i}^{r}}{1+\alpha_{1} y_{1}^{r}+\alpha_{2} y_{2}^{r}}$ and $p^{12}\left(y_{1}, y_{2}\right)=\frac{1}{1+\alpha_{1} y_{1}^{r}+\alpha_{2} y_{2}^{r}}, i \in[1,2]$. It is straightforward to verify that this CSF satisfies all axioms 1-4. Q.E.D. 


\section{Proof of theorem 3}

For convenient notation let us introduce the following family of functions. $H^{N}\left(y_{N}\right)=1$, $H^{S}\left(y_{S}\right)=f^{S}\left(y_{S}\right) /\left(1-f^{S}\left(y_{S}\right)\right)$ where $f^{S}: \mathrm{R}_{+}^{|S|} \rightarrow[0,1]$ is an arbitrary function used in axiom 3a. It is possible then to rewrite axiom $3 \mathrm{a}$ as (A4)

$$
p^{S}\left(y_{N}\right)=H^{S}\left(y_{S}\right) \sum_{\substack{T \subseteq \Omega \\ S \subseteq T}} p^{T}\left(y_{N}\right), \forall S \subseteq \Omega
$$

because $\sum_{\substack{T \subseteq \Omega \\ S \subseteq T}} p^{T}\left(y_{N}\right)=1-\sum_{\substack{T \subseteq \Omega \\ S \subseteq T}} p^{T}\left(y_{N}\right)$ due to axiom 1a. Equation (A4) defines the probability distribution $p^{S}\left(y_{N}\right), S \subseteq \Omega$ recursively. Iterating every probability $p^{T}\left(y_{N}\right)$ on the right hand side of (A4) we obtain (A5).

$$
p^{S}\left(y_{N}\right)=H^{S}\left(y_{S}\right) \cdot\left[\sum_{\substack{A \subseteq \Omega \\ S \subset A}} H^{A}\left(y_{A}\right) \sum_{\substack{B \subseteq \Omega \\ A \subset B}} H^{B}\left(y_{B}\right) \ldots H^{N}\left(y_{N}\right)\right] \cdot p^{N}\left(y_{N}\right), \forall S \subseteq \Omega
$$

Since equation (A5) holds for any element of $\Omega$ it is possible to sum (A5) over all elements of $\Omega$. On the left hand side of equation (A5) we then obtain $\sum_{S \subseteq \Omega} p^{S}\left(y_{N}\right)=1$ due to

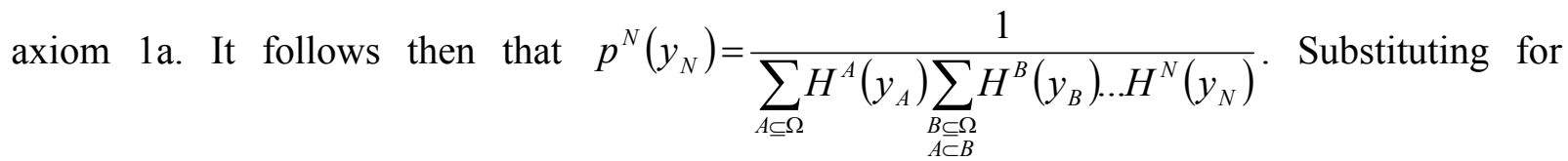
$p^{N}\left(y_{N}\right)$ in (A5) we finally obtain $p^{S}\left(y_{N}\right)=\frac{H^{S}\left(y_{S}\right) \sum_{\substack{A \subseteq \Omega \\ S \subset A}} H^{A}\left(y_{A}\right) \sum_{\substack{B \subseteq \Omega \\ A \subset B}} H^{B}\left(y_{B}\right) \ldots H^{N}\left(y_{N}\right)}{\sum_{\substack{A \subseteq \Omega \\ A}} H^{A}\left(y_{A}\right) \sum_{\substack{B \subseteq \Omega \\ A \subset B}} H^{B}\left(y_{B}\right) \ldots H^{N}\left(y_{N}\right)}, \forall S \subseteq \Omega$. It is straightforward to verify that this CSF satisfies all axioms 1a-3a and therefore these axioms are both necessary and sufficient. Q.E.D. 